\title{
ARTICLE \\ Infections in the management of rheumatic diseases: An update
}

\author{
S A Botha-Scheepers, MD, PhD (The Netherlands); B Sarembock, MB ChB, FCP (SA), Cert Rheumatology (SA) \\ Division of Rheumatology, Department of Medicine, Faculty of Health Sciences, Groote Schuur Hospital and University of Cape Town, South Africa
}

Corresponding author: S A Botha-Scheepers (stella.botha@uct.ac.za)

Patients with inflammatory rheumatic conditions have an increased risk of infection. While this could be the result of the underlying disease, it may also be caused by the use of immunosuppressive therapies, which are needed to treat these disorders. An increasing number of patients with rheumatoid arthritis or other rheumatic diseases are using biologic therapies (biologics) in addition to the synthetic diseasemodifying anti-rheumatic drugs. The side-effects and complications of these relatively new agents are unknown to many specialists (outside of rheumatology) and general practitioners. This article highlights updates on the most important infections encountered in the daily management of patients with rheumatic diseases and discusses how these may be prevented.

S Afr Med J 2015;105(12):1076. DOI:10.7196/SAMJ.2015.v105i12.10220

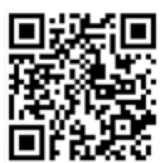

\section{Viral infections}

Patients with inflammatory rheumatic conditions have an increased risk of infection, including viral infections. ${ }^{[1]}$ Rheumatological manifestations, such as arthritis, are also relatively common occurrences with viral infections.

\section{Hepatitis B}

The prevalence of hepatitis B virus (HBV) in rheumatic patients has recently been reported at $\sim 3 \%$ in a large cross-sectional study on comorbidities, which included almost 4000 rheumatoid arthritis (RA) patients at 17 centres worldwide. ${ }^{[2]}$ The prevalence differed according to geographical area - more infections in Asia and fewer in Europe and the USA. However, it demonstrates this common problem in daily rheumatological practice owing to the risk of hepatitis $B$ reactivation through immunosuppressive therapy.

According to the American College of Rheumatology (ACR) guidelines and the views of experts, RA patients should be screened for HBV before initiation of methotrexate, leflunomide and biologic therapies. ${ }^{[3]}$ Patients with chronic hepatitis B should receive treatment with oral antivirals before starting therapy. Those with significant liver damage should, however, not receive these immunosuppressive drugs.

There are no guidelines with regard to the use of synthetic disease-modifying anti-rheumatic drugs (sDMARDs) and biologics in patients with a history of hepatitis B infection (hepatitis B core antibody positive). A significant population of those who were exposed to HBV, have cleared the virus. Depending on the prevalence of chronic HBV infection, the prevalence of past $\mathrm{HBV}$ infection varies between $5 \%$ and $80 \%$ worldwide. In the literature on haematology (lymphomas), rituximab-containing chemotherapy regimens lead to reactivation in $3-25 \%$ of patients with past HBV infection. ${ }^{[4]}$ As data are limited, it is not clear if pre-emptive antiviral prophylaxis is needed in a rheumatological setting, but recently a large prospective study in $>1200$ RA patients demonstrated a risk of reactivation in cases of previous HBV infection (15\%) treated with biologics. ${ }^{[5]}$ The majority were treated with anti-tumour necrosis factor (anti$\mathrm{TNF}$ ), but rituximab and other biologics were also used. It was reassuring that there were no observed cases of $\mathrm{HBV}$ reactivation during biologic treatment. This confirms the results of previous studies that the risk of HBV reactivation in patients with rheumatic diseases and a history of HBV infection treated with anti-TNF and rituximab is very low. ${ }^{[6]}$ However, more studies should be done to assess the need for pre-emptive antiviral prophylaxis in patients with past HBV infection. In practice, regular monitoring for viral reactivation during therapy, particularly in patients lacking evidence of immunity ( $\mathrm{HBs} \mathrm{Ab}$ negative), is necessary. If the transaminase levels are increased, HBV DNA should be checked and antiviral therapy initiated if reactivation occurs.

\section{Hepatitis C}

Hepatitis C virus (HCV) infection is a major global health problem, with a prevalence of $2.8 \%$. Universal screening for individuals between the ages of 45 and 65 years is recommended by the US Centers for Disease Control and Prevention (CDC) ${ }^{[7]} \mathrm{HCV}$ can be associated with rheumatic syndromes, such as the potentially serious rheumatic complication of HCV-associated cryoglobulinaemic vasculitis (CV).

To treat $\mathrm{HCV}$, interferon alpha (IFNa)-based regimens can achieve viral clearance in about $50 \%$ of patients, but it has many side-effects (e.g. cytopenias, depression, flu-like symptoms) and renal impairment is a contraindication. New oral direct-acting antivirals (DAAs) are being introduced into clinical practice. A prospective cohort study on treatment with a combination of first-generation DAAs with PegIFNa and ribavirin in 30 patients with severe refractory CV, who were not responding to standard treatment with antiviral therapy, was published recently. ${ }^{[8]}$ After 1 year of treatment, $67 \%$ of these patients showed a clinical response, with total clearance of the virus. However, there were many side-effects, such as cytopenias and infections.

Fortunately, the newer DAAs, which target specific proteins such as non-structural protein $3 \mathrm{~b} / 4 \mathrm{~A} / 5 \mathrm{~A}$, will it is hoped signal the end of interferon use in HCV patients in the future. ${ }^{[9,10]}$ These new drugs could possibly cure the disease in $>90 \%$ of patients, with fewer side-effects. They are extremely expensive and only administered to patients with the highest risk of complications, such as those with $\mathrm{CV}$ with end-organ manifestations. ${ }^{[1]}$ Despite the high cost, they are considered to be cost-effective. ${ }^{[12]}$ Data on these new drugs in patients with rheumatic diseases are confined to a few case reports, but several trials are currently underway. 
Data on the use of biologics in patients with hepatitis $\mathrm{C}$ infections are scarce. Biologics are contraindicated in cases of acute or chronic $\mathrm{HCV}$ infection with significant liver damage, as progression of $\mathrm{HCV}$ has been described in such patients who receive biologics. In RA patients with chronic HCV infection without significant liver damage, rituximab and anti-TNF (the ACR suggests etanercept as the preferred anti-TNF) may be used, but monitoring of serum transaminases is advised.

\section{Herpes zoster}

Reactivation of the latent varicella zoster virus causes herpes zoster (HZ), a painful vesicular skin rash that could be complicated by chronic pain and post-herpetic neuralgia. In patients with rheumatic disease, the incidence of $\mathrm{HZ}$ is almost 12 times higher in RA, 20 times higher in systemic lupus erythematosus (SLE) and 45 times higher in Wegener's granulomatosus. ${ }^{[13]}$ This increased risk of HZ is caused by the underlying disease, especially in those receiving immunosuppressive therapy. ${ }^{[14]}$ A recent review showed that there was some increased risk with the use of biologics (anti-TNF) and sDMARDs, ${ }^{[15]}$ but that high-dose corticosteroids and cyclophosphamide led to the highest increase in risk.

\section{HIV}

HIV can cause several rheumatological manifestations, including an inflammatory polyarthritis mimicking RA. Therefore, an HIV test may be useful in patients presenting with inflammatory arthritis. HIV can also result in several challenges in the management of patients with rheumatic diseases, who are also HIV-positive, especially if not on antiretroviral treatment. Data on the safety of immunosuppressive drugs in HIV-positive patients are limited, but there have been reports on the use of sDMARDs and TNF- $\alpha$ inhibitors to treat RA or other inflammatory conditions in HIV infection. ${ }^{[16,17]}$ In general, these reports suggest that DMARDs such as methotrexate and antiTNF are tolerated well by HIV-positive patients, provided that the patients are on an effective antiretroviral regimen before the start of therapy.

\section{Vaccinations}

According to the latest recommendations by the CDC, immunocompromised patients such as those with rheumatic diseases should receive killed (pneumococcal, annual intramuscular influenza and hepatitis B) and recombinant (human papillomavirus $(\mathrm{HPV})$ ) vaccines before starting an sDMARD or a biologic. ${ }^{[18]}$ It also recommends that, if not previously done, vaccination with pneumococcal, intramuscular influenza, HBV and HPV vaccines should be done in patients already taking an sDMARD or a biologic. All vaccines should be given based on age and risk, and physicians should refer to vaccine instructions and CDC recommendations for details about dosing and timing. Importantly, both the pneumococcal polysaccharide vaccine (PPSV23) and the 13-valent conjugate pneumococcal vaccine (PCV13) should be given. In non-immunised patients, one should start with the PCV13, then wait for 2 months, and thereafter administer the PPSV23 vaccine. In patients $<65$ years of age, a repeat dose of the PPSV 23 should be given after 5 years. Live virus vaccines (such as the live attenuated influenza vaccine; measles, mumps, rubella) should not be administered to immunosuppressed patients with rheumatic diseases.

The COMORA study showed how often patients with rheumatic diseases were being vaccinated. ${ }^{[2,19]}$ This study was performed at 17 centres worldwide, where $25 \%$ of RA patients had received an influenza vaccination in the past year, $17 \%$ a pneumococcal vaccination in the past 5 years, and only $10 \%$ both vaccinations. It is not known how often South African (SA) patients with rheumatic diseases are vaccinated, but this shows that clinicians, especially rheumatologists, must do more to ensure adequate vaccination in these patients.

\section{Efficacy and safety of the $\mathrm{HZ}$ vaccine}

In immunocompetent individuals the risk of $\mathrm{HZ}$ can be reduced by 50 $70 \%$ with a live attenuated vaccine. ${ }^{[20]}$ According to the ACR guidelines, the live $\mathrm{HZ}$ vaccine may be given to all patients with rheumatic diseases before starting immunosuppressive therapy. ${ }^{[3]}$ Once on such therapy, patients may receive the vaccine if treated with sDMARDs (monoor combination therapy), but it is contraindicated in those receiving biologics or high-dose steroids (prednisone $>20 \mathrm{mg} /$ day) owing to a concern of developing a varicella infection from the vaccine virus strain.

Recently a study on the association between $\mathrm{HZ}$ vaccination and the risk of $\mathrm{HZ}$ infection in patients with immune-mediated diseases such as RA, psoriatic arthritis and ankylosing spondylitis was published. ${ }^{[21]}$ Of the 465000 patients, $<5 \%$ were vaccinated against HZ. The vaccine, however, showed a similar efficacy in healthy individuals, with a decreased risk of $\mathrm{HZ}$ (adjusted hazard ratio (HR) 0.61 ) and an $80 \%$ decrease in post-herpetic neuralgia. Interestingly, in the 633 patients who were treated with biologics $(87 \%$ on antiTNF), there were no cases of varicella or HZ. The ACR published an update suggesting that it may be reasonable to discontinue a biologic for a period, administer the $\mathrm{HZ}$ vaccine and then resume the biologic after 30 days.

\section{Tuberculosis}

The 2012 ACR guidelines recommend screening for latent tuberculosis (TB) infection (LTBI) in all patients with rheumatic diseases being considered for biologic therapy, regardless of the presence of risk factors. ${ }^{[3]}$ Doctors should assess the patient's medical history to identify other risk factors (such as smoking and corticosteroid use) for TB and perform the tuberculin skin test (TST) or interferon-gammarelease assays (IGRAs) initially in all patients starting biologic agents. Patients should have a chest radiograph and, if suggestive of active $\mathrm{TB}$, a sputum examination should be done to test for the presence of active TB. In a high-prevalence TB setting such as SA, these screening tests are not always reliable and there is little consensus on the most appropriate test, ${ }^{[22]}$ but a TST and/or an IGRA (if deemed appropriate by the clinician) may be performed. If both tests are used, they should be done at the same time, as TST could boost IGRA responses, which may confound the interpretation of the results.

Chemoprophylactic drugs for LTBI may be either isoniazid (INH) for 9 months or rifampicin combined with INH for 3 months. Treatment with biologic agents can be initiated or resumed after 1 month of LTBI treatment and after completion of the treatment of active TB. Some experts and studies suggest that non-TNF biologic therapies, such as rituximab and abatacept, are safer and better choices as firstline biologics in patients in high-burden settings. ${ }^{[23]}$

It remains uncertain how to manage RA patients with a negative initial screening for TST or IGRA on chronic biologic therapy. In low-prevalence TB settings patients may not need further evaluation in the absence of risk factors and/or clinical suspicion for TB. However, patients may have false-negative TST or IGRA results owing to immunosuppression. Therefore, a negative TST or IGRA does not exclude the possibility that a patient has LTBI. An updated consensus statement by the ACR recommends that repeat screening should be considered in areas of high TB risk populations or in the case of potential TB exposure. Recent studies, however, have shown that TB conversions may occur during anti-TNF therapy in $\sim 10 \%$ of RA patients. ${ }^{[24]}$ It is unclear 
if these conversions always represent 'true conversions'. More data are needed to make recommendations regarding rescreening in the absence of definite TB exposure.

\section{Serious infections in RA}

It is not well understood why patients with RA have an increased prevalence of other serious infections, particularly pulmonary, urinary tract, skin, and joint infections. Factors that may contribute include immunosuppression by the disease, treatment of the disease, presence of inflammatory lung disease, or factors associated with disability and immobility. Compared with the increased risk of infection associated with the use of corticosteroids and biologics, the sDMARDs do not appear to be associated with such a risk.

Biologics have been used in RA for almost 15 years and rheumatologists have gained much experience with these drugs. A large number of randomised controlled trials have been published on their efficacy and safety, and data exist from national registries worldwide. An important question that often arises with regard to a patient taking these drugs is: 'What is the risk of a serious infection when on biologic therapy compared with being on sDMARDs?'

A number of meta-analyses have assessed this risk. ${ }^{[25-27]}$ Recently, the largest meta-analysis to date ( $>42000$ patients) assessed the rate of serious infection in RA. ${ }^{[28]}$ The authors found that if the rate of serious infection on standard doses of biologic therapy (with or without sDMARDs) is compared with that of sDMARD monotherapy, the risk is increased by $30 \%$ (odds ratio 1.31 ). It is important to understand what this means in absolute numbers. The absolute annual risk for serious infections when using sDMARD monotherapy is $2 \%$. Therefore, $2 \%$ of the RA population on sDMARDs experience a serious infection every year. The risk was increased by $30 \%$ when using biologic therapy, resulting in an absolute risk of $2.6 \%$. The absolute increase in the number of serious infections compared with traditional DMARDs was 6/1 000 patients per year for standard-dose biologic therapy with or without traditional DMARDs. One would therefore have to treat $1000 \mathrm{RA}$ patients with biologic therapy to cause 6 more serious infections compared with traditional DMARDs.

\section{Serious infections in SLE}

The risk of serious infections in cases of SLE was reviewed recently. ${ }^{[29]}$ In SLE, $20-30 \%$ of deaths are caused by infections. In a US study, the rate of serious infections in SLE was $~ 10.8 / 100$ patient-years, which is approximately 5 times higher than in RA patients. ${ }^{[30]}$ In lupus nephritis (LN) this risk was almost 12 times higher than in RA patients, i.e. 23.9/100 patient-years. Men and black patients with SLE were found to have an increased risk of serious infection. In this study, traditional immunosuppressive therapy in SLE, such as azathioprine, mycophenolate mofetil and cyclophosphamide, showed a $10 \%$ increased risk (HR 1.11), but not in the LN group (HR 0.92). With the use of corticosteroids there was an increased risk of $>50 \%$ (HR 1.51) in SLE and almost 25\% in LN patients (HR 1.23). Interestingly, the rate of serious infections was decreased by almost $30 \%$ in SLE and $20 \%$ in LN when using chloroquine. The most common serious infections in SLE are bacterial, such as pneumonia, urinary tract infection, opportunistic infection, sepsis and skin infections. ${ }^{[31]}$ These studies confirm the importance of limiting the dose of corticosteroids in SLE patients.

\section{Conclusion}

Patients with rheumatic diseases have an increased risk of infections owing to their condition and immunosuppressive therapies. This article highlights the latest research findings with regard to the most important infections encountered in the daily management of patients with rheumatic diseases. This knowledge can be used when balancing the potential harm and the clinically important benefits of sDMARDs and biologics and will assist patients and their physicians to make evidence-based decisions.

\section{References}

1. Falagas ME, Manta KG, Betsi GI, Pappas G. Infection-related morbidity and mortality in patients with connective tissue diseases: A systematic review. Clin Rheumatol 2007;26(5):663-670. [http://dx.doi. org/10.1007/s10067-006-0441-9]

2. Dougados M, Soubrier M, Antunez A, et al. Prevalence of comorbidities in rheumatoid arthritis and evaluation of their monitoring: Results of an international, cross-sectional study (COMORA). Ann Rheum Dis 2014;73(1):62-68. [http://dx.doi.org/10.1136/annrheumdis-2013-204223]

3. Singh JA, Furst DE, Bharat A, et al. 2012 update of the 2008 American College of Rheumatology recommendations for the use of disease-modifying antirheumatic drugs and biologic agents in the treatment of rheumatoid arthritis. Arthritis Care Res 2012; 64(5):625-639. [http://dx.doi.org/10.1002/ acr.21641]

4. Kim HY, Kim W. Chemotherapy-related reactivation of hepatitis B infection: Updates in 2013. World J Gim HY, Kim W. Chemotherapy-related reactivation of hepatitis B infection: Updates
Gastroenterol 2014;20(40):14581-14588. [http://dx.doi.org/10.3748/wjg.v20.i40.14581]

Gastroenterol 2014;20(40):14581-14588. [http://dx.doi.org/10.3748/wjg.v20.i40.14581]
5. Barone M, Notarnicola A, Lopalco G, et al. Safety of long-term biologic therapy in rheumatologic patients with a previously resolved hepatitis B viral infection. Hepatology 2015;62(1):40-46. [http:// dx.doi.org/10.1002/hep.27716]

6. Caporali R, Bobbio-Pallavicini F, Atzeni F, et al. Safety of tumor necrosis factor alpha blockers in hepatitis B virus occult carriers (hepatitis B surface antigen negative/anti-hepatitis B core antigen positive) with rheumatic diseases. Arthritis Care Res (Hoboken) 2010;62(6):749-754. [http://dx.doi. org/10.1002/acr.20130]

7. Smith BD, Morgan RL, Beckett GA, et al. Recommendations for the identification of chronic hepatitis C virus infection among persons born during 1945 - 1965. MMWR Recomm Rep 2012;61(RR-4):1-32.

8. Saadoun D, Resche Rigon M, Pol S, et al. PegIFNa/ribavirin/protease inhibitor combination in severe hepatitis C virus-associated mixed cryoglobulinemia vasculitis. J Hepatol 2015;62(1):24-30. [http:// hepatitis C virus-associated mixed cry
dx.doi.org/10.1016/j.jhep.2014.08.015]

9. Kowdley KV, Lawitz E, Poordad F, et al. Phase $2 \mathrm{~b}$ trial of interferon-free therapy for hepatitis $\mathrm{C}$ virus 9. Kowdley KV, Lawitz E, Poordad F, et al. Phase 2b trial of interferon-free therapy for hepatitis
genotype 1. N Engl J Med 2014;370(3):222-232. [http://dx.doi.org/10.1056/NEJMoa1306227]

10. Afdhal N, Zeuzem S, Kwo P, et al. Ledipasvir and sofosbuvir for untreated HCV genotype 1 infection. N. Afdhal N, Zeuzem S, Kwo P, et al. Ledipasvir and sofosbuvir for untreated HCV genotype
N Engl J Med 2014;370(20):1889-1898. [http://dx.doi.org/10.1056/NEJMoa1402454]

N Engl J Med 2014;370(20):1889-1898. [http://dx.doi.org/10.1056/NEJMoal402454]
1. AASLD/IDSA HCV Guidance Panel. Hepatitis C guidance: AASLD-IDSA recommendations for 1. AASLD/IDSA HCV Guidance Panel. Hepatitis C guidance: AASLD-IDSA recommendations for
testing, managing, and treating adults infected with hepatitis C virus. Hepatology 2015;62(3):932-954. [http://dx.doi.org/10.1002/hep.27950]

12. Najafzadeh M, Andersson K, Shrank WH, et al. Cost-effectiveness of novel regimens for the treatment of hepatitis C virus. Ann Intern Med 2015;17;162(6):407-419. [http://dx.doi.org/10.7326/M14-1152]

13. Cohen JI. Clinical practice: Herpes zoster. N Engl J Med 2013;369(3):255-263. [http://dx.doi.org/10.1056/ NEJMcp1302674]

14. Strangfeld A, Listing J, Herzer P, et al. Risk of herpes zoster in patients with rheumatoid arthritis treated with anti-TNF-alpha agents. JAMA 2009;301(7):737-744. [http://dx.doi.org/10.1001/jama.2009.146]

15. Westra J Rondaan $C$ van Assen $S$, Bijl M. Vaccination of patients with autoimmune influmm r.sta rheumatic diseases. Nat Rev Rheumatol 2015;11(3):135-145. [http://dx.doi.org/10.1038/nrrheum.2014.206] 6. Kaur PP, Chan VC, Berney SN. Successful etanercept use in an HIV-positive patient with rheumatoid arthritis. J Clin Rheumatol 2007;13(2):79-80. [http://dx.doi.org/10.1097/01.rhu.0000260411.75599.39]
Cepeda EJ, Williams FM, Ishimori ML, Weisman MH, Reveille JD. The use of anti-tumour necrosis 7. Cepeda EJ, Williams FM, Ishimori ML, Weisman MH, Reveille JD. The use of anti-tumour necrosis
factor therapy in HIV-positive individuals with rheumatic disease. Ann Rheum Dis 2008;67(5):710factor therapy in HIV-positive individuals with rhe
712. [http://dx.doi.org/10.1136/ard.2007.081513]

712. [http://dx.doi.org/10.1136/ard.2007.081513]
8. Kim DK, Bridges CB, Harriman KH, Centers for Disease Control and Prevention (CDC), Advisory Committee on Immunization Practices (ACIP), ACIP Adult Immunization Work Group. Advisory committee on immunization practices recommended immunization schedule for adults aged 19 years or older - United States, 2015. MMWR Morb Mortal Wkly Rep 2015;64(4):91922. [http://dx.doi. org/10.7326/m14-2755]

19. Hmamouchi I, Winthrop K, Launay O, Dougados M. Low rate of influenza and pneumococcal vaccine coverage in rheumatoid arthritis: Data from the international COMORA cohort. Vaccine 2015;33(12):1446-1452. [http://dx.doi.org/10.1016/j.vaccine.2015.01.065]

20. Schmader KE, Levin MJ, Gnann JW, et al. Efficacy, safety, and tolerability of herpes zoster vaccine in persons aged 50 - 59 years. Clin Infect Dis 2012;54(7):922-928. [http://dx.doi.org/10.1093/cid/cir970]

21. Zhang J, Xie F, Delzell E, et al. Association between vaccination for herpes zoster and risk of herpes Zhang J, Xie F, Delzell E, et al. Association between vaccination for herpes zoster and risk of herpes
zoster infection among older patients with selected immune-mediated diseases. JAMA 2012;308(1):43zoster infection among older patients with selected

22. Hodkinson B, van Duuren E, Pettipher C, Kalla A, South African Rheumatism and Arthritis Hodkinson B, van Duuren E, Pettipher C, Kalla A, South African Rheumatism and Arthritis
Association. South African recommendations for the management of rheumatoid arthritis: An algorithm Association. South African recommendations for the management of rheumatoid arthritis: An algorithm
for the standard of care in 2013. S Afr Med J 2013;103(8):576-585. [http://dx.doi.org/10.7196/samj.7047] 3. Tikly M, Hodkinson B, Dheda K. Biologic therapy for rheumatoid arthritis in developing countries - a place for non-TNF inhibitors as first-line treatment? Rheumatology 2015;54(2):208-209. [http:// dx.doi.org/10.1093/rheumatology/keu040]

24. Hatzara C, Hadziyannis E, Kandili A, et al. Frequent conversion of tuberculosis screening tests during anti-tumour necrosis factor therapy in patients with rheumatic diseases. Ann Rheum Dis 2014;74(10):1848-1853.

25. Leombruno JP, Einarson TR, Keystone EC. The safety of anti-tumour necrosis factor treatments in rheumatoid arthritis: Meta and exposure-adjusted pooled analyses of serious adverse events. Ann Rheum Dis 2009;68(7):1136-1145. [http://dx.doi.org/10.1136/ard.2008.091025]

26. Salliot $C$, Dougados $M$, Gossec L. Risk of serious infections during rituximab, abatacept and anakinra Salliot C, Dougados M, Gossec L. Risk of serious infections during rituximab, abatacept and anakinra
treatments for rheumatoid arthritis: Meta-analyses of randomised placebo-controlled trials. Ann treatments for rheumatoid arthritis: Meta-analyses of randomised place
Rheum Dis 2009;68(1):25-32. [http://dx.doi.org/10.1136/ard.2007.083188]

27. Thompson AE, Rieder SW, Pope JE. Tumor necrosis factor therapy and the risk of serious infection and malignancy in patients with early rheumatoid arthritis: A meta-analysis of randomized controlled and malignancy in patients with early rheumatoid arthritis: A meta-analysis of rando
trials. Arthritis Rheum 2011;63(6):1479-1485. [http://dx.doi.org/10.1002/art.30310]

28. Singh JA, Cameron C, Noorbaloochi S, et al. Risk of serious infection in biological treatment of patients with rheumatoid arthritis: A systematic review and meta-analysis. Lancet 2015;386(9990):258-265. [http://dx.doi.org/10.1016/S0140-6736(14)61704-9]

29. Widdifield J, Bernatsky S. Connective tissue diseases: The burden of serious infections in SLE. Nat Rev Rheumatol 2015;11(6):326-327. [http://dx.doi.org/10.1038/nrrheum.2015.55]

30. Feldman $\mathrm{CH}$, Hiraki LT, Winkelmayer WC, et al. Serious infections among adult Medicaid beneficiaries with systemic lupus erythematosus and lupus nephritis. Arthritis Rheumatol 2015;67(6):1577-1585. [http://dx.doi.org/10.1002/art.39070]

31. Tektonidou MG, Wang Z, Dasgupta A, Ward MM. Burden of serious infections in adults with systemic lupus erythematosus: A national population-based study, 1996 - 2011. Arthritis Care Res 2015;67(8):1078-1085. [http://dx.doi.org/10.1002/acr.22575] 